\title{
Evaluation of detector dynamic range in the x-ray exposure domain in mammography: A comparison between film-screen and flat panel detector systems
}

\author{
Virgil N. Cooper III, ${ }^{\text {a) }}$ Thomas Oshiro, Christopher H. Cagnon, \\ and Lawrence W. Bassett \\ David Geffen School of Medicine at UCLA, Department of Radiological Sciences, \\ 200 UCLA Medical Plaza, Los Angeles, California 90095 \\ Tyler M. McLeod-Stockmann \\ University of Michigan Medical School, C5124 Medical Science Building I, 1301 Catherine Street, \\ Ann Arbor, Michigan 48109 \\ Nikita V. Bezrukiy \\ David Geffen School of Medicine at UCLA, Department of Radiological Sciences, \\ 200 UCLA Medical Plaza, Los Angeles, California 90095
}

(Received 27 August 2002; accepted for publication 15 July 2003; published 17 September 2003)

Digital detectors in mammography have wide dynamic range in addition to the benefit of decoupled acquisition and display. How wide the dynamic range is and how it compares to film-screen systems in the clinical x-ray exposure domain are unclear. In this work, we compare the effective dynamic ranges of film-screen and flat panel mammography systems, along with the dynamic ranges of their component image receptors in the clinical x-ray exposure domain. An ACR mammography phantom was imaged using variable mAs (exposure) values for both systems. The dynamic range of the contrast-limited film-screen system was defined as that ratio of mAs (exposure) values for a $26 \mathrm{kVp} \mathrm{Mo} / \mathrm{Mo}(\mathrm{HVL}=0.34 \mathrm{~mm} \mathrm{Al})$ beam that yielded passing phantom scores. The same approach was done for the noise-limited digital system. Data from three independent observers delineated a useful phantom background optical density range of 1.27 to 2.63 , which corresponded to a dynamic range of $2.3 \pm 0.53$. The digital system had a dynamic range of $9.9 \pm 1.8$, which was wider than the film-screen system $(p<0.02)$. The dynamic range of the film-screen system was limited by the dynamic range of the film. The digital detector, on the other hand, had an estimated dynamic range of 42 , which was wider than the dynamic range of the digital system in its entirety by a factor of 4 . The generator/tube combination was the limiting factor in determining the digital system's dynamic range. (C) 2003 American Association of Physicists in Medicine.

[DOI: $10.1118 / 1.1606450]$

Key words: dynamic range, flat panel, digital mammography, ACR phantom

\section{INTRODUCTION}

In film-screen mammography, the range of $\mathrm{x}$-ray exposures exiting the breast may be large relative to the useful dynamic range of the film. Exposures exiting the least and most attenuating portions of the breast, respectively, may be sufficiently high or low to yield optical densities (ODs) in the shoulder or toe of the film characteristic curve. Thus, a lack of signal contrast results in these areas. To circumvent this occurrence, more penetrating beams may be used to effectively compress the dynamic range of information exiting the breast, thus putting that exposure information in the domain of the linear portion of the film's characteristic curve. However, this comes with the consequence of an overall decreased contrast in the image as radiographic (subject) contrast and display contrast are inexorably coupled. As a result, there have been numerous investigations focused on equalization of the x-ray exposure information presented to the screen-film system. ${ }^{1-5}$ These investigations concentrated on modulating the intensities incident on the breast by placing anatomically appropriate filters in the beam to reduce the intensity of the beam for the less attenuating portions of the breast, while leaving the intensity corresponding to the more attenuating portions of the breast undisturbed. Thus, a more uniform intensity pattern was presented to the film-screen cassette whereby the range of exposures exiting the breast was sufficiently narrow to be mapped into the useful optical density range of the film. Postprocessing solutions have also been attempted to overcome the limitations of film. ${ }^{6}$

The inexorably coupled acquisition and display and the dynamic range limitations of film are not present in digital mammography. Once images are acquired using digital technology, those images may be arbitrarily windowed, leveled, or otherwise processed for more adequate display on either softcopy (monitors) or hardcopy (film) devices. ${ }^{7,8}$ Furthermore, digital mammography systems are thought to have a much wider dynamic range than conventional film-screen systems, ${ }^{9-12}$ but the quantification of how wide that range is in clinical practice is not well documented. The dynamic ranges of a digital flat panel imager and a typical film-screen system for mammography have, respectively, been reported 
at 200:1 and 25-50:1. ${ }^{12}$ It is thought that digital mammography systems yield fewer repeats in imaging compared to film-screen systems because of this widened dynamic range.

This study was borne out of a question as to how wide a range of technique factors ( $\mathrm{mAs}$ ) could be used to produce acceptable digital images as compared to film-screen systems (ignoring dose constraints). As will be shown, the exposure factors for the typical exam in our clinic are very similar to those for the American College of Radiology (ACR) accreditation phantom. Thus, to answer this question, we perform a simple study using the ACR accreditation phantom. To be sure, there are known questions about the suitability of the ACR phantom and its use for digital mammography systems ${ }^{13}$ but currently, it is the standardized phantom that is effectively required by law for any institution performing either digital or screen-film screening mammography in the United States.

In effect, there are two different questions that may be asked. How wide is the dynamic range of the image receptor in clinical practice as determined by imaging the ACR phantom? How wide is the dynamic range of the system as a whole in clinical practice as determined by imaging the ACR phantom? It is questioned whether the dynamic range of the film-screen unit as a system, including the x-ray tube and generator, versus the film-screen receptor alone (under our processing conditions) are effectively the same. That is, it is hypothesized that for a given $\mathrm{kVp} /$ target/filter combination, there is a low mAs value below which the ACR phantom image would be unacceptable. Is there a high $\mathrm{mAs}$ value above which the ACR image would also be unacceptable? Will the generator permit such a value? The same questions are asked for the digital system.

Whether it would ever be beneficial to expose the detector, and hence the breast, at exposure levels significantly higher than those associated with the lowest mAs value that yields an acceptable image under normal conditions is unclear. But, a case may be made that although the increased numbers of quanta result in a higher dose, those quanta would yield better lesion signal-to-noise ratio and hence, aid in lesion detectability, particularly for digital detectors with smaller pixels. ${ }^{14}$ If the doses were not prohibitive and if the generator/tube combination were of sufficient power, it seems plausible that "high dose diagnostic mammography" could potentially aid in radiologist characterization of lesions in diagnostic exams and decrease the number of unnecessary biopsies. Of course, this would require further clinical studies to determine this.

In the case of film, it is effectively the detector contrast derived from the characteristic curve that defines the useful mAs values that can be used in the phantom imaging. In the case of the digital system, it is the noise level relative to the signal at the low end and the saturation point at the high end that would yield the range of mAs values that produce suitable ACR phantom images.

\section{MATERIALS AND METHODS}

The comparison of the dynamic ranges of the film-screen and digital systems begins with a statistical model. We (null) hypothesize that in our clinical setting, the useful dynamic range of film-screen system and the dynamic range of the digital system are the same. We seek to reject that null hypothesis and show that in the clinical setting, the dynamic range of our flat panel digital mammography system is wider than our conventional film-screen system. The hypothesis testing may be given as

$$
\begin{aligned}
& H_{0}: \mathrm{DR}_{\text {film }}=\mathrm{DR}_{\text {digital }}, \\
& H_{a}: \mathrm{DR}_{\text {film }}<D R_{\text {digital }},
\end{aligned}
$$

where $H_{0}$ is the null hypothesis; $H_{a}$ is the alternate hypothesis; $\mathrm{DR}_{\text {film }}$ is the film-screen system dynamic range; and $\mathrm{DR}_{\text {digital }}$ is the digital system dynamic range. A one-tailed t-test is used to determine whether to reject the null hypothesis $(\alpha=0.05)$.

For a clinical comparison, we choose to use x-ray beams that are representative of those that are used in the clinical setting. A random sampling of 85 craniocaudad mammographic images from different patients showed that $\mathrm{Mo} / \mathrm{Mo}$ spectra were used $73 \%$ of the time (mean $26.22 \mathrm{kVp} \pm 1.37$ $\mathrm{kVp}$ ). The average compressed breast thickness of these patients was $4.8 \mathrm{~cm} \pm 1.2 \mathrm{~cm}$. Since these data are consistent with the American College of Radiology (ACR) mammographic phantom imaging in our clinic, and since, by definition of being in compliance with the Mammography Quality Standards Act (MQSA), all sites performing mammography must have one, we use the ACR phantom and a $26 \mathrm{kVp}$ $\mathrm{Mo} / \mathrm{Mo}$ beam for this analysis.

While ascertaining the exposure range-dependent contrast in a film-screen system and the exposure range-dependent $\mathrm{x}$-ray quantum-limited noise behavior in a digital system are somewhat straightforward, the extent to which these concepts correspond to the observer-defined usefulness of the images is not clear. Therefore, small observer studies are performed to evaluate the usefulness of these images. For completeness, however, we include standard sensitometric measurements to illustrate the contrast behavior for our film in our processing conditions. We also include exposuredependent signal and noise transfer characteristics of the digital system.

\section{A. Screen-film system sensitometry}

Three films were exposed to a calibrated sensitometer and processed with our clinical film processor. The steps of the sensitometric strip were known to vary by a factor of $2^{1 / 2}$ $\approx 1.41$ in exposure (light output). The resulting mean optical densities, OD, were plotted against relative exposure, $X$, and fit with a third-order polynomial within the diagnostic region of OD,

$$
\mathrm{OD}(X)=\sum_{i=0}^{3} a_{i} X^{i}
$$

where $a_{i}$ is the $i$ th fitting coefficient. From this fit, exposure contrast, $C$, was then calculated as

$$
C(X)=\frac{d}{d X}[\mathrm{OD}(X)]
$$


The exposure contrast was then replotted against optical density to show the contrast as a function of the optical density.

\section{B. Screen-film system observer study}

To the best of our knowledge, there exist no definitive data detailing the lower and upper end points of optical density that are clinically useful and applicable to an individual site's film use and processing conditions. Thus, a small-scale observer study was used to delineate the useful optical density range, and hence, the dynamic range. The standardized ACR accreditation phantom (Gammex RMI, Middleton, WI) was imaged with our clinical film-screen system (Fuji AD Medium screen, Fuji AD-M film, Fujifilm Medical Systems, USA, Inc., Stamford, CT) and a clinical mammography unit (GE DMR, General Electric Medical Systems, Milwaukee, WI) using a $26 \mathrm{kVp} \mathrm{Mo/Mo} \mathrm{beam}(\mathrm{HVL}=0.34 \mathrm{~mm} 99.99 \%$ pure A1). The linearity between exposure and mAs was confirmed. Three sets of ten images were obtained at incremental mAs (and by linearity, exposure) values. Each image in each set of ten images was assigned a random number and the images were placed in separate folders in a random fashion (i.e., no order from light to dark, etc.). The images were given one set at a time to three independent readers. The readers consisted of two physicists and one radiologist. Using ACR guidelines, the readers scored the images for fibers, speck groups, and masses under appropriate masked clinical viewing conditions. After the readers returned their respective sets along with the score sheets to the study coordinator, the sets were exchanged and the readers received a different set of images and score sheets. This was done again after the readers read their second sets. The resulting mean phantom scores (and standard deviation) for each reader were tabulated as a function of the mAs used to produce the images. The average of all observer scores was also plotted against the individual mAs values. For each observer, the useful dynamic range of the film-screen system used in our clinic was taken to be that ratio of exposures (average mAs values) that composed the thresholds of the continuous exposure region of passing MQSA scores of 4 fibers, 3 speck groups, and 3 masses. That is, for each observer, the low mAs threshold (and low OD threshold) was the point at which the average of the three sets of phantom scores first passed the MQSA values as below this the images were too light and the information appeared in the toe of the film characteristic curve. Likewise, the high mAs threshold (and high OD threshold) was the point at which the average of the three sets of phantom scores last passed the MQSA values. Above this, the images were too dark and the information appeared in the shoulder of the characteristic curve. After the mAs thresholds were clearly delineated for each observer, the dynamic range of the film was defined as

$$
D_{\text {film }}=\frac{Q_{\max }}{Q_{\min }},
$$

where $Q_{\max }$ and $Q_{\min }$ are, respectively, the highest and lowest mAs values (averaged over the observers) at which the phantom images received a passing score. The uncertainty in the low and high mAs thresholds, $\sigma_{\min }, \sigma_{\max }$, was calculated as the standard deviation of the observer thresholds. For a function, $f$, of $N$ independent variables, the variance, $\sigma_{f}^{2}$, in that function may be approximated as

$$
\sigma_{f\left(x_{1}, \ldots, x_{N}\right)}^{2}=\sum_{n=1}^{N}\left(\frac{\partial f\left(x_{1}, \ldots, x_{N}\right)}{\partial x_{n}}\right)^{2} \sigma_{x_{n}}^{2},
$$

where $\sigma_{x_{n}}^{2}$ is the variance in the $n$th independent variable. The uncertainty in the dynamic range estimate was found by applying Eq. (5) to Eq. (4).

To delineate the useful optical density range for our film in our processing conditions, the six threshold images were examined for the optical density in the center of the phantom. The low OD threshold and the high OD threshold were calculated as the average ODs (in the background of the phantom) of the three low mAs and high mAs images, respectively.

\section{Digital system signal and noise evaluation}

Under similar conditions as the film-screen system, the ACR phantom was imaged with a $26 \mathrm{kVp} \mathrm{Mo/Mo} \mathrm{beam}$ $(\mathrm{HVL}=0.37 \mathrm{~mm}$ 99.99\% pure $\mathrm{A} 1)$ on a state-of-the-art digital mammography system (GE Senographe, 2000D, General Electric Medical Systems, Milwaukee, WI). Again, the linearity between exposure and mAs was confirmed. The phantom was imaged with a wide range of mAs values, and hence, exposure values. The signal and noise values were tabulated as a function of mAs (exposure) for both the manufacturer-identified "RAW" (linear) and "PROCESSED" (log) data.

As film-screen systems have a film-associated toe and shoulder that effectively determine the dynamic range of those systems, digital systems have a "noise floor" and "saturation ceiling" that determine where the system can be operated without a significant loss of x-ray signal statistics. As we showed optical density and contrast as a function of exposure with the film-screen system, we begin here by examining the exposure-dependent pixel signal and noise characteristics with the digital system. The low mAs (low exposure) point at which the system became $x$-ray quantum limited defined the "floor" for the measurement of dynamic range. This is readily done using the PROCESSED data. Determining the "ceiling" for the dynamic range description requires an estimation and extrapolation of signal and noise values to the saturation point based on the acquired RAW data which are known to be linear. ${ }^{15}$

Pixel signal means and standard deviations were collected from $0.35 \mathrm{~cm}^{2}$ regions of interest (ROIs) within the center of the ACR phantom images. The signal values were plotted against mAs. Both the manufacturer-defined "RAW" linear gain/offset/dead pixel-corrected 14 bit data (hereafter referred to as "raw") and the "PROCESSED" 12 bit logtransformed data (hereafter referred to as "processed") were examined. However, for conciseness, only the processed data will be shown in this manuscript. 
The processed data are the data that are viewed by clinicians. Since the processed data from this system are given in a negative mode (low exposures equate to high numbers), the polarity of the data was inverted. The mean pixel value as a function of exposure, $X$, was then given by

$$
S(X)=S_{\max }-S_{\log }(X),
$$

where $S(X)$ is the inverted $\log$ signal, $S_{\max }$ is the maximum digital grayscale number (4095 for this 12 bit data), and $S_{\log }(X)$ is the native processed data from this particular system. The signal transfer curve for this inverted log image data was parametrized as

$$
S(X)=m \ln (X)+b,
$$

where $m$ and $b$ are fitting coefficients that are supplied by a logarithmic fit of the ROI average signal against exposure. Given the signal transfer curve, the expected behavior of the noise was ascertained. Applying Eq. (5) to Eq. (7) was used to give the expected variance in the signal data, which is given by

$$
\sigma_{S(X)}^{2}=\frac{m^{2} \sigma_{X}^{2}}{X^{2}}
$$

Assuming that the variance in exposure was due to quantum statistics and that other sources of uncertainty in the x-ray beam (e.g., polychromaticity) were negligible, then the exposure variance is proportional to the exposure (Poisson statistics) and Eq. (8) may be reduced to a proportionality,

$$
\sigma_{X}^{2} \propto \frac{m^{2}}{X} .
$$

Thus, the exposure range over which the pixel variance exhibited a linear relationship with the reciprocal of exposure was used to define the x-ray quantum-limited exposure region of operation. As will be shown, the low exposure end point at which the system became x-ray quantum limited was clearly identified. The upper exposure end point at which the system saturates is found by extrapolating the signal and noise as a function of exposure on the raw data which exhibit a well-behaved linear response.

\section{Digital system observer study}

As for the screen-film observer study, an observer study was also performed for the digital system with two physicists and one radiologist scoring the ACR phantom. Three sets of ACR phantom images were acquired with each set being imaged with a $26 \mathrm{kVp} \mathrm{Mo/Mo} \mathrm{beam,} \mathrm{in} \mathrm{a} \mathrm{random} \mathrm{order} \mathrm{from}$ 10 to $450 \mathrm{mAs}$. The $450 \mathrm{mAs}$ value was the maximum allowed by the generator. Using ACR scoring guidelines, the readers reviewed the images in a random order of $\mathrm{mAs}$ and scored the images for fibers, speck groups, and masses under clinical softcopy viewing conditions. The reviewers were given no guidelines regarding the amount of windowing and leveling that they could perform. The resulting mean phantom scores (and standard deviation) for each reader were tabulated as a function of the mAs used to produce the im-

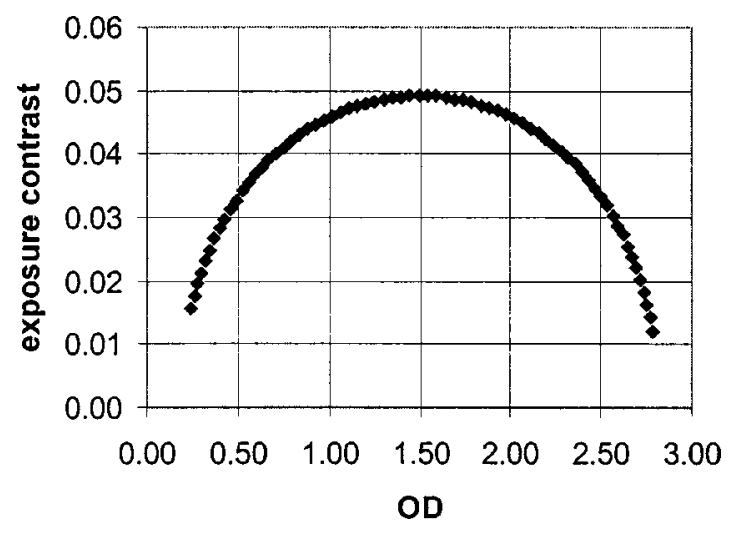

FIG. 1. The exposure contrast, computed from the first derivative of optical density with respect to exposure, is plotted against optical density.

age. The average of all observer scores were also plotted against the individual $\mathrm{mAs}$ values. For each observer, the useful dynamic range of the digital system used in our clinic was taken to be that ratio of exposures (average mAs values) that composed the thresholds of the MQSA-required score of 4 fibers, 3 speck groups, and 3 masses. That is, for each observer, the low mAs threshold was the lowest mAs within the continuous domain of MQSA passing phantom scores, as below this the images were too noisy. Likewise, the high mAs threshold was the point at which the average of the three sets of phantom scores last passed the MQSA values.

Given the upper and lower mAs (exposure) end points for the digital system, the dynamic range was defined as

$$
D_{\text {digital }}=\frac{Q_{\text {max, digital }}}{Q_{\text {min, digital }}},
$$

where $Q_{\text {max,digital }}$ and $Q_{\text {min,digital }}$ refer to the maximum and minimum $\mathrm{mAs}$ values over which the system yielded passing ACR phantom scores.

\section{RESULTS AND DISCUSSION}

\section{A. Screen-film system sensitometry}

Figure 1 shows the exposure contrast curve for the film and processing conditions at UCLA. As expected, the contrast is diminished at low and high optical densities.

\section{B. Screen-film system observer study}

Figure 2 shows the average of all nine phantom scores against mAs. For the $26 \mathrm{kVp} \mathrm{Mo} / \mathrm{Mo}$ beam $(\mathrm{HVL}=0.34 \mathrm{~mm}$ 99.99\% pure $\mathrm{Al}$ ), the input exposure to the phantom and exit exposure from the phantom were 9.0 and $0.24 \mathrm{mR} / \mathrm{mAs}$, respectively. The entrance skin exposure (ESE) to mean glandular dose (MGD) conversion factor was $172 \mathrm{mrad} / \mathrm{R}$, thus yielding an mAs-dose conversion factor of $1.55 \mathrm{mrad} / \mathrm{mAs}$. The optical densities of the individual films were later measured in the center of the phantom images and the average phantom scores were replotted against optical density in Fig. 3. Although somewhat sparsely sampled in the "sweet spot" of the characteristic curve, the center of the passing area is where the center of the phantom had an optical density of 


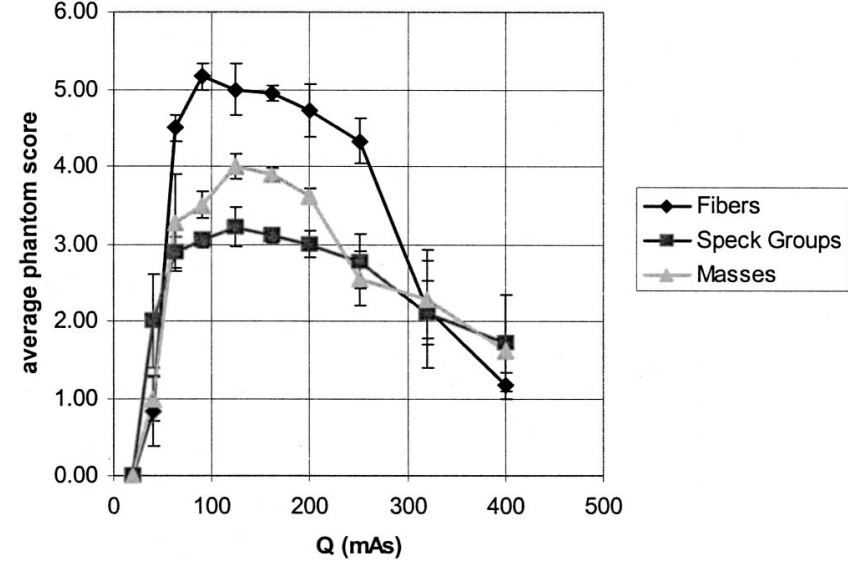

FIG. 2. The phantom scores averaged over observers (error bars: $\pm \sigma$ ) are plotted against mAs. Speck group detection was consistently the hardest of the detection tasks. Phantom exit exposure factor: $0.24 \pm 5$ $\times 10^{-3} \mathrm{mR} / \mathrm{mAs}$ at breast support located $64 \mathrm{~cm}$ from focal spot. mAs-dose conversion factor: $1.55 \mathrm{mrad} / \mathrm{mAs}$.

1.9. Based on these detection data, we have since modified our automatic exposure control to give a background OD of 1.9. Previously, it was at 1.6 , presumably based on the chemistry and film manufacturers' input. This baseline optical density change represents a $12 \%$ increase in mean glandular dose.

Based on Eq. (4), the film dynamic range, defined by the exposure end points ( $\mathrm{mAs}$ end points averaged over observers) where passing ACR phantom scoring criteria is achieved, is approximately 2.3 . The uncertainty in that measurement of 0.53 was calculated by applying Eq. (5) to Eq. (4) for the individual observer data. Referring to Fig. 3, the average measured phantom scores (across all observations) against mAs shows that the closest mAs end point images that defined the dynamic range corresponded to background optical densities of 1.27 for the $90 \mathrm{mAs}$ image $(22 \mathrm{mR}$ at the breast support) and 2.63 for the $200 \mathrm{mAs}$ image $(48 \mathrm{mR}$ at the breast support). These data are in good agreement with ACR-published optical density limits on contrast degradation

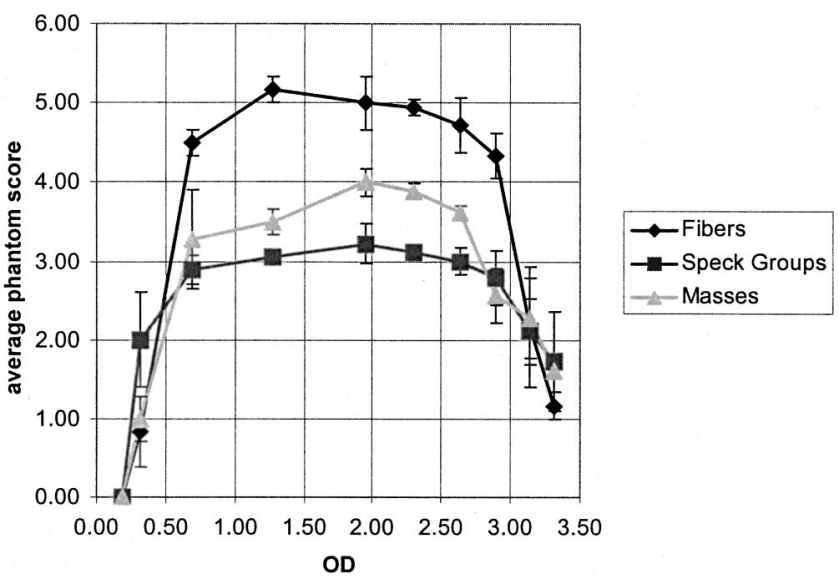

FIG. 3. The average phantom scores (error bars: $\pm \sigma$ ) are plotted against OD. The center of the passing region is near an OD of 1.9 .

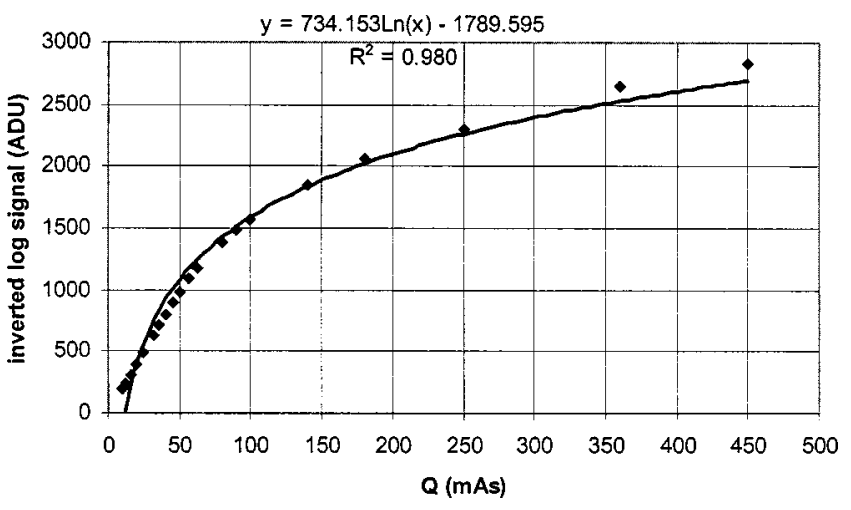

FIG. 4. The converted transfer curve is presented along with a relatively poor $\log$ fit. Phantom exit exposure factor: $0.23 \pm 5 \times 10^{-3} \mathrm{mR} / \mathrm{mAs}$ at breast support located $64 \mathrm{~cm}$ from focal spot.

which suggests diminished contrast for films with optical densities below 1.0-1.25 and above 2.5-3.0. ${ }^{16}$

Based on these data, speck group imaging was consistently the most challenging of the imaging tasks. This was consistent across all observers. To note, if a one-tailed $95 \%$ confidence test were applied in this analysis, as can be seen by examining the means and standard deviations in Figs. 2 and 3 , the acceptable mAs region of operation would have remained unchanged for fibers and masses. However, for speck groups, there would have been no acceptable region of operation. This further suggests that the detection/scoring criteria for speck groups is much more stringent than for masses and fibers.

\section{Digital system signal and noise evaluation}

Figure 4 shows the 12 bit processed transfer curve data as transformed by Eq. (6). For the $26 \mathrm{kVp} \mathrm{Mo/Mo} \mathrm{beam} \mathrm{(HVL}$ $=0.37 \mathrm{~mm} 99.99 \%$ pure $\mathrm{Al}$ ), the input exposure to the phantom and exit exposure from the phantom were 8.4 and 0.23 $\mathrm{mR} / \mathrm{mAs}$, respectively. The ESE to MGD conversion factor was $185 \mathrm{mrad} / \mathrm{R}$, thus making the $\mathrm{mAs}$ to MGD conversion factor $1.55 \mathrm{mrad} / \mathrm{mAs}$. Although the data are known to be logarithmic, adding a logarithmic trend line in Fig. 4 yielded somewhat of a poor fit. Figure 5 shows the pixel variance plotted against the reciprocal of mAs. There is an apparent dual-component transfer function of the flat panel imager. The pixel variance increases in a linear-like fashion up to 0.025 reciprocal mAs (from 450 down to $40 \mathrm{mAs}$ ). At that point, the pixel variance decreases with reciprocal $\mathrm{mAs}$ in a polynomial fashion. This was further investigated by separating the data into two components corresponding to mAs values up to $40 \mathrm{mAs}$ (9.2 $\mathrm{mR}$ at the breast support), and those above $40 \mathrm{mAs}$. For reference, the ACR phantom is imaged at $110 \mathrm{mAs}$ ( $25 \mathrm{mR}$ at the breast support) in our clinic. Figure 6 shows the $\log$ of the pixel variance against the $\log$ of the mAs for 10-40 mAs. The slope of 0.595 indicates that the variance tracks as $\mathrm{mAs}^{0.595}$. This is consistent with neither a linear detector nor a logarithmic detector as the expected relationship would be $\mathrm{mAs}^{1.0}$ or $\mathrm{mAs}^{-1.0}$, respectively. 


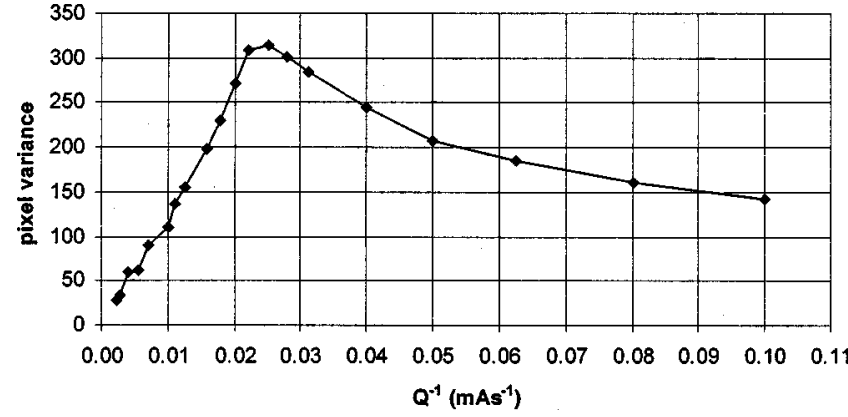

FIG. 5. The pixel variance is plotted against reciprocal mAs. These data indicate a bimodal behavior of the imager where the pixel variance increases with increasing exposure in the low exposure (mAs) region, and where it decreases with increasing exposure in the high exposure region. Phantom exit exposure factor: $0.23 \pm 5 \times 10^{-3} \mathrm{mR} / \mathrm{mAs}$ at breast support located 64 $\mathrm{cm}$ from focal spot.

At low reciprocal $\mathrm{mAs}$ (high $\mathrm{mAs}$ ) values, the expected linear relationship between pixel variance and reciprocal mAs results. Figure 7 shows the $\log$ of the pixel variance against the $\log$ of the mAs for 40-450 mAs. The slope of -1.011 is in good agreement with the expected power relationship of $\mathrm{mAs}^{-1.0}$. Based on the analysis of noise, the inverted logarithmic transfer curve shown in Fig. 4 was replotted in a more limited mAs domain, 40-450 mAs. Figure 8 shows the resultant fit. As the data show, at the higher exposure levels, the expected logarithmic relationship between signal level and mAs is shown with excellent precision. The pixel variance follows the expected relationship as is shown in Fig. 7. At the lower exposure levels, a more linear relationship is evident; however, the pixel variance does not follow the expected linear relationship with mAs. The nature of these behaviors is unclear. However, further study of bit limitation and relative influence of additive and quantum noise in this region may shed light on this relationship. Based on these behaviors, the log-processed data indicate that the detector becomes x-ray quantum limited at $40 \mathrm{mAs}(9.2 \mathrm{mR}$ at the breast support).

While not shown, this noise floor was confirmed by evaluating the raw linear data. The detector saturation limit was found using the raw linear data to be $1884 \mathrm{mAs}(433 \mathrm{mR}$ at

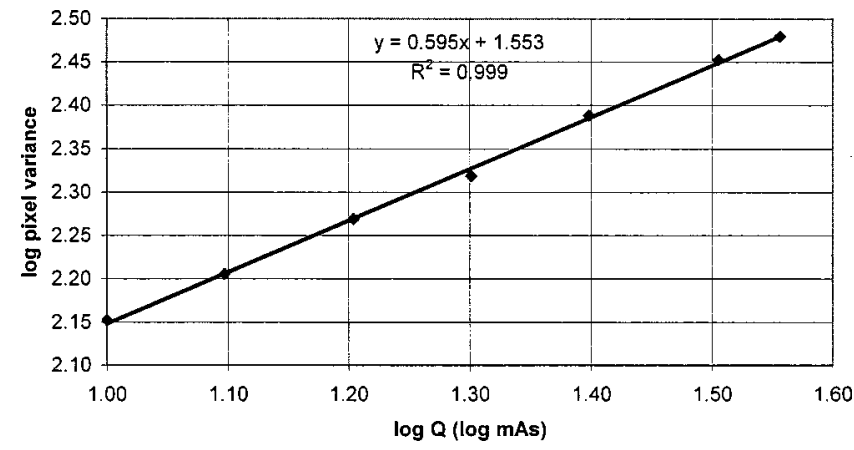

FIG. 6. The log of pixel variance is plotted against the $\log$ of mAs for the low mAs region. The fit indicates that the pixel variance is proportional to $\mathrm{mAs}^{0.595}$. This is consistent with neither a $\log$ nor a linear transfer curve. Phantom exit exposure factor: $0.23 \pm 5 \times 10^{-3} \mathrm{mR} / \mathrm{mAs}$ at breast support located $64 \mathrm{~cm}$ from focal spot.

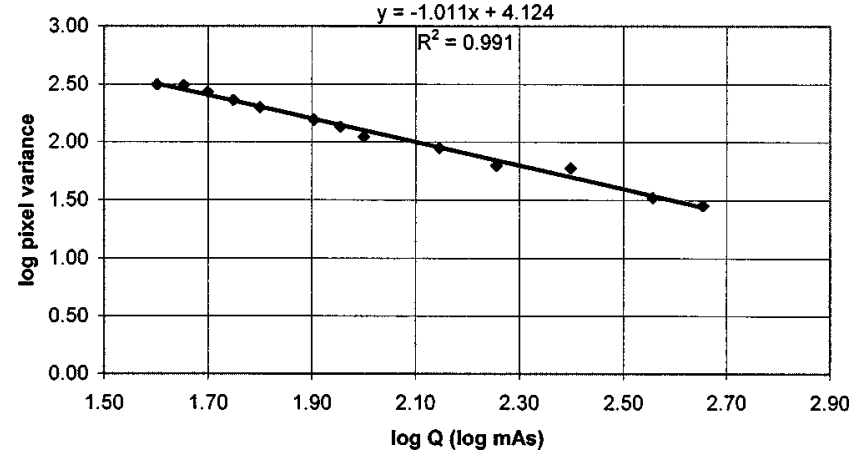

FIG. 7. The log of pixel variance is plotted against the log of mAs for the high mAs region. The fit indicates that the pixel variance is proportional to $\mathrm{mAs}^{-1.011}$. This is consistent with a logarithmic transfer curve. Phantom exit exposure factor: $0.23 \pm 5 \times 10^{-3} \mathrm{mR} / \mathrm{mAs}$ at breast support located $64 \mathrm{~cm}$ from focal spot.

the breast support) by way of extrapolation of signal and noise as a function of exposure to the exposure level such that the signal plus twice the standard deviation of the signal would be equivalent to the maximum digital signal value of $2^{14}-1=16383$. Thus, the detector alone had an estimated exposure range of $1884 / 40(\sim 47)$ over which it is $\mathrm{x}$-ray quantum limited without significant saturation. The digital mammography system, however, cannot achieve such an $\mathrm{mAs}$, as the maximum that the generator allowed was 450 mAs.

\section{Digital system observer study}

Figure 9 shows the average phantom scores against mAs for the three observers. As expected, the detectability of the phantom structures is significantly degraded at low mAs values due to poor signal-to-noise characteristics in the images. As was found in Huda et al., for increasing mAs values, there was little change in absolute phantom scores beyond a certain exposure (mAs). However, the data indicate lower intraobserver as well as interobserver variability with increasing mAs, but this will need to be studied further before any definitive conclusions are made. Saturation, which would not be expected at these mAs values, is not a factor in the data. Thus, the ACR phantom images, from a scoring

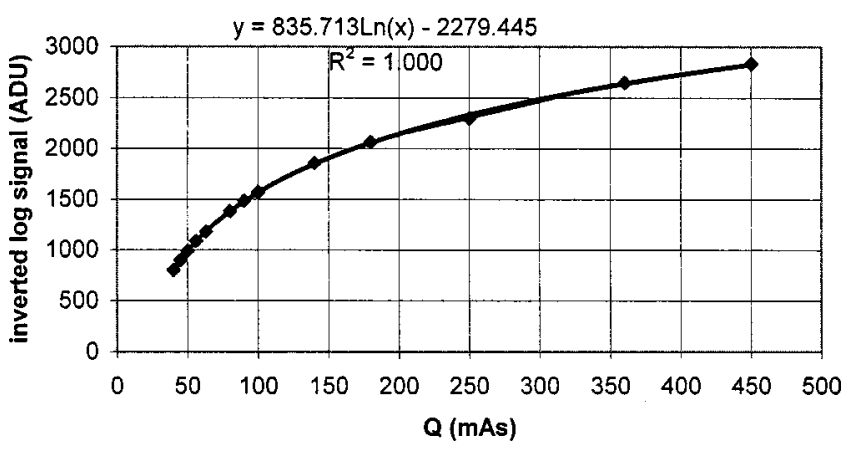

FIG. 8. The signal transfer curve is replotted in a limited domain (the quantum-limited domain). The data exhibit the expected log fit to a high degree. Phantom exit exposure factor: $0.23 \pm 5 \times 10^{-3} \mathrm{mR} / \mathrm{mAs}$ at breast support located $64 \mathrm{~cm}$ from focal spot. 


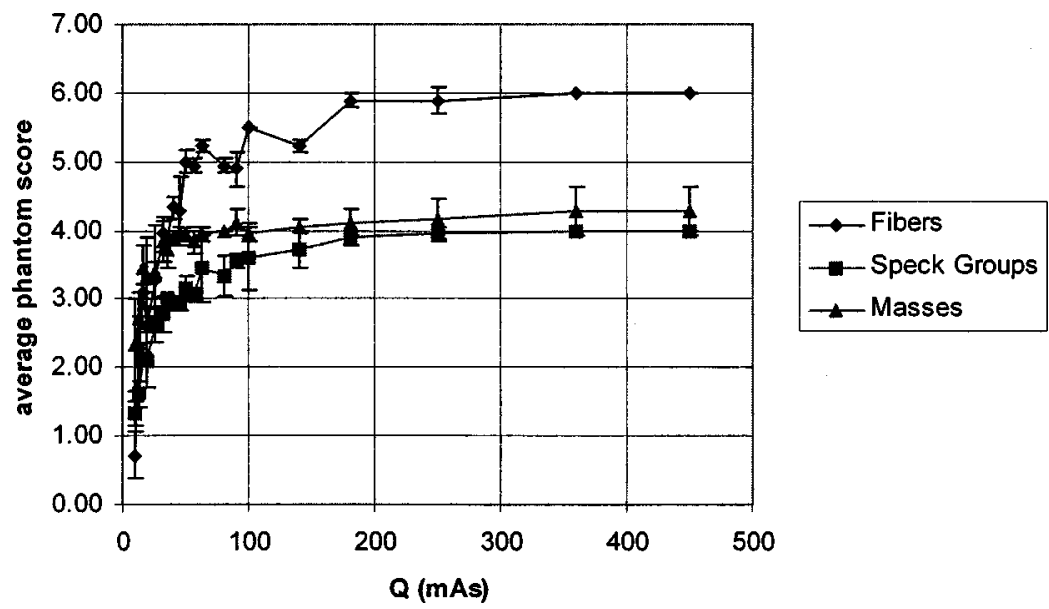

FIG. 9. The phantom scores averaged over observers (error bars: $\pm \sigma$ ) are plotted against $\mathrm{mAs}$ for the digital system. Speck group detection was consistently the hardest of the detection tasks. Phantom exit exposure factor: $0.23 \pm 5 \times 10^{-3} \mathrm{mR} / \mathrm{mAs}$ at breast support located $64 \mathrm{~cm}$ from focal spot. mAs-dose conversion factor: $1.55 \mathrm{mrad} / \mathrm{mAs}$.

perspective and not a dosimetric perspective, were acceptable at $450 \mathrm{mAs}$, the highest level allowed by the tube/ generator system.

Figure 10 shows the phantom scores plotted against $\mathrm{mAs}$ from the lowest $\mathrm{mAs}$ point up to clinical imaging $\mathrm{mAs}$ point. The system first passes the 4, 3, 3 scoring criteria at approximately $45 \mathrm{mAs}$, which is slightly above but consistent with the point at which the detector became x-ray quantum limited. This may be pure coincidence and needs to be investigated further. The system maintains the MQSA passing criteria up to the mAs limit of 450 . Based on averaging the lower and upper mAs end points over observers, the digital mammography system has a dynamic range of $9.9 \pm 1.8$.

\section{CONCLUSIONS AND FURTHER DISCUSSIONS}

The film-screen system as a whole and the image receptor had equivalent dynamic ranges of 2.3 as defined in this work. That is, the film/processing itself was the limiting factor in determining the dynamic range of the film-screen system. For the digital system, the limiting factor was not the image receptor. It was the tube/generator combination. However, both the dynamic range of the digital system (9.9) and the dynamic range of the digital detector (estimated at 42) are much wider than that of the film-screen system ( $p$ $<0.02)$. Thus, the null hypothesis is rejected.
It has been suggested that the decoupled acquisition contrast and display contrast of digital systems makes for more efficient imaging for women with dense breasts as harder spectra (i.e., more penetrating spectra produced by higher $\mathrm{kVp}$ and/or different target/filter combinations) could be used to produce the images that could subsequently be windowed/ leveled or otherwise processed. Before a harder beam is used, however, it may be advantageous to understand exactly what the expected range (ratio) of exposures exiting dense breasts under current clinical conditions (i.e., softer beams) really is. A softer beam does produce more radiographic (subject) contrast, and as such, can lead to greater differences in lesion and background signals. Thus, although contrast is somewhat arbitrary in digital systems, it may nevertheless be advantageous to use softer beams to produce images with higher lesion signal-to-noise ratios; that is, given that the dynamic range of the detector is sufficiently wide enough to encompass that range and given that the dose is acceptable.

As the digital system data also indicated, although the number of detected speck groups only slightly improved with increasing $\mathrm{mAs}$ in a particular range, the precision of speck group detection was markedly improved as a function of the increased numbers of quanta (e.g., 450 compared to $100 \mathrm{mAs}$ ). Whether this translates into a meaningful clinical scenario is unknown, but a case may be made that it is

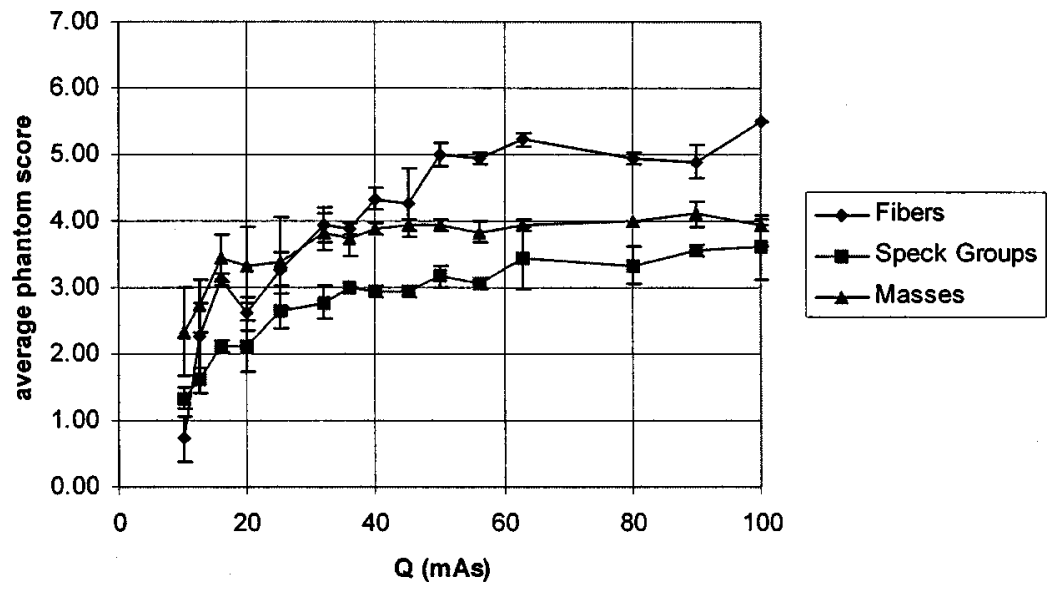

FIG. 10. The phantom scores averaged over observers (error bars: $\pm \sigma$ ) are plotted against mAs in the low mAs domain. Speck group detection was consistently the hardest of the detection tasks. Phantom exit exposure factor: $0.23 \pm 5 \times 10^{-3} \mathrm{mR} / \mathrm{mAs}$ at breast support located $64 \mathrm{~cm}$ from focal spot. mAs-dose conversion factor: $1.55 \mathrm{mrad} / \mathrm{mAs}$.

Medical Physics, Vol. 30, No. 10, October 2003 
worthwhile to investigate the feasibility of acquiring diagnostic images at increased doses (e.g., in this case 4.5 times the dose). While a dose increase of 4.5 times is probably not warranted in screening mammography, it may yield some benefit in diagnostic mammography if these ACR phantom results are at all translatable to the clinical imaging scenario.

In helping to formulate imaging protocols for the clinic, one of our constraints should be that the images should be acquired such that the pertinent tissue signals are acquired in the quantum-limited domain. Thus, for this digital system, the ratio between the exposures exiting the least and most attenuating projection volumes of the breast should be lower than the useful dynamic range of the detector (i.e., 42). With such a wide range of exposures, potentially, even siliconeaugmented breasts might be able to be imaged such that some relevant tissue signal is detected in the projection path of the silicone implant. In a cursory random sampling, the average exposure dynamic range of information exiting the silicone-augmented breast was $18.8 \pm 6.4$ for the CC images and 21.0 \pm 8.6 for the MLO images. (These ranges are well below the dynamic range of the digital detector, but above the range of the system as a whole.) However, it will have to be studied further to see if meaningful image information in silicone implants can be acquired in a dosimetrically acceptable manner.

\section{SUMMARY}

We have measured the dynamic range of a state-of-the-art digital mammography system and a film-screen system in the clinical exposure domain. Both signal transfer metrics and observer studies were used to detail the behaviors of the two systems. The dynamic ranges were defined by the ability of each system to yield MQSA passing ACR phantom images as a function of exposure (mAs) at a particular $\mathrm{kVp} /$ target/filter combination. The film/processing was the limiting factor in determining the dynamic range for the filmscreen system. The generator/tube combination was the limiting factor for the digital system. The data indicate that the digital system can handle an exposure range (ratio) at least four times wider than the film-screen system used in our clinic. The digital detector, alone, can handle an exposure range of almost 20 times wider than the film-screen receptor/processing scenario.

\section{ACKNOWLEDGMENTS}

The authors are grateful for the support of the California Breast Cancer Research Program (5TB-0070) and GE Medi- cal Systems. We are also grateful to Dr. Carolyn KimmeSmith for useful discussions on exposure information exiting the breast.

${ }^{a)}$ Author to whom all correspondence should be addressed; electronic mail: vcooper@mednet.ucla.edu

${ }^{1}$ M. M. Goodsitt, H. P. Chan, B. Liu, S. V. Guru, A. R. Morton, S. Keshavmurthy, and N. Petrick, "Classification of compressed breast shapes for the design of equalization filters in x-ray mammography," Med. Phys. 25, 937-948 (1998).

${ }^{2}$ J. M. Sabol, I. C. Soutar, and D. B. Plewes, "Practical application of a scan-rotate equalization geometry to mammography," Med. Phys. 23, 1987-1996 (1996).

${ }^{3}$ J. M. Sabol and D. B. Plewes, "Analytical description of the high and low contrast behavior of a scan-rotate geometry for equalization mammography," Med. Phys. 23, 887-898 (1996).

${ }^{4}$ J. M. Sabol, I. C. Soutar, and D. B. Plewes, "A method for practical equalization mammography of the radiographically dense breast," Radiographics 15, 1191-1202 (1995).

${ }^{5}$ J. M. Sabol, K. K. Tam, and D. B. Plewes, "Rotary scanning equalization radiography: An efficient geometry for equalization mammography," Med. Phys. 21, 1523-1533 (1994).

${ }^{6}$ A. P. Stefanoyiannis, L. Costaridou, P. Sakellaropoulos, and G. Panayiotakis, "A digital density equalization technique to improve visualization of breast periphery in mammography," Br. J. Radiol. 73, 410-420 (2000).

${ }^{7}$ E. D. Pisano, E. B. Cole, B. M. Hemminger, M. J. Yaffe, S. R. Aylward, A. D. Maidment, R. E. Johnston, M. B. Williams, L. T. Niklason, E. F. Conant, L. L. Fajardo, D. B. Kopans, M. E. Brown, and S. M. Pizer, "Image processing algorithms for digital mammography: A pictorial essay," Radiographics 20, 1479-1491 (2000).

${ }^{8}$ R. Sivaramakrishna, N. A. Obuchowski, W. A. Chilcote, G. Cardenosa, and K. A. Powell, "Comparing the performance of mammographic enhancement algorithms: A preference study," AJR, Am. J. Roentgenol. 175, 45-51 (2000).

${ }^{9}$ H. Liu, L. L. Fajardo, J. R. Barrett, and R. A. Baxter, "Contrast-detail detectability analysis: Comparison of a digital spot mammography system and an analog screen-film mammography system," Acad. Radiol. 4, 197-203 (1997).

${ }^{10}$ W. Zhao, J. Law, D. Waechter, Z. Huang, and J. A. Rowlands, "Digital radiology using active matrix readout of amorphous selenium: Detectors with high voltage protection," Med. Phys. 25, 539-549 (1998).

${ }^{11}$ A. D. A. Maidment, R. Hahrig, and M. J. Yaffe, "Dynamic range requirements in digital mammography," Med. Phys. 20, 1621-1633 (1993).

${ }^{12} \mathrm{~S}$. Muller, "Full field digital mammography designed as a complete system," Eur. J. Radiol. 31, 25-34 (1999).

${ }^{13}$ W. Huda, A. M. Sajewicz, K. M. Ogden, E. M. Scalzetti, and D. R. Dance, "How good is the ACR accreditation phantom for assessing image quality in digital mammography?," Acad. Radiol. 9, 764-772 (2002).

${ }^{14}$ V. N. Cooper III, J. M. Boone, and J. A. Seibert, "A lesion detectability simulation method for digital x-ray imaging," Med. Phys. 27, 66-74 (2000).

${ }^{15}$ S. Vedantham, A. Karellas, S. Suryanarayan, D. Albagli, S. Han, E. J. Tkaczyk, C. E. Landberg, P. R. Granfors, I. Levis, C. J. D’Orsi, and R. E. Hendrick, "Full breast digital mammography with an amorphous siliconbased flat panel detector: Physical characteristics of a clinical prototype," Med. Phys. 27, 558-567 (2000).

${ }^{16}$ R. E. Hendrick et al., "ACR mammography quality control manual," 1999, p. 142. 